\title{
Patent Valuation Under Spatial Point Processes with Delayed and Decreasing Jump Intensity
}

\author{
Roy Cerqueti ${ }^{a}$, Marco Ventura ${ }^{b, *}$ \\ ${ }^{a}$ Department of Economics and Law \\ University of Macerata, Italy - roy.cerqueti@unimc.it \\ ${ }^{b}$ ISTAT \\ Italian National Institute of Statistics, Rome, Italy - mventura@istat.it
}

September 9, 2014

\begin{abstract}
This article is set within the real options approach applied to patent valuation. Such evaluation is based on the knowledge of the impact of some events on the underlying state, which is modeled in discrete time as a spatial point process, i.e. both size and time of the jumps can be treated as random variables. This assumption allows us to improve upon the current theory of patent valuation in some respects. In particular, the propagation of the jumps from the economic environment to the patent value is not restricted to be immediate, but can occur with a random delay and with varying intensity, depending on the time to maturity. These actual features lead to a more general formula for patent value, that may give rise to a non trivial difference not accounted for in the existing literature.
\end{abstract}

Keywords: Patent value; jump; Stochastic Point Process; Real Option. JEL Classification: L00; O34; C65.

\section{Introduction}

This paper provides a contribution to the literature on patents valuation. Assigning economic value to patents is relevant for patentees and at social level. Probably due to this twofold relevance, the economic valuation of patents has attracted much attention and efforts among researchers, insomuch that extensive documentation has been provided since the second half of the '80s. In this paper we move from the common assumption that a patent is an option over a technology so that modeling

\footnotetext{
${ }^{*}$ Corresponding author: ISTAT, Italian National Institute of Statistics. P.zza Dell'Indipendenza 4, 00185, Rome, Italy. Tel.: +39(0)6 4673 3665; Fax: +39(0)6 233232 419. Email: mventura@istat.it
} 
can proceed as a derivative contract where the patent value is linked to the properties of the underlying asset. Indeed, a patent gives the holder the exclusive right (option) on an invention. The right can be renewed to the expiration date $T$ or be dropped before the statutory limit, if the holder decides not to pay the renewal fee. The real options approach is based on the definition of an underlying asset the evolution of which drives the patent value. In agreement with industrial economics theory, the underlying asset is given by the cash flow associated to the patent. Specifically, the fluctuations in the underlying asset represent the quantitative translation of the qualitative characteristics of the economic environment. Under this assumption, the underlying is able to capture the uncertainty affecting supply and demand of goods, the production of which is entitled through patent ownership, research programs from other firms, the attainment of patents, the changes in the regulatory environment, etc. These factors are not associated to a continuous time horizon, but rather they occur at discrete points in time, causing the underlying state to undergo a jump (for instance, it is quite unrealistic to assume that changes in the laws protecting patents occur continuously). This fact implies the presence of jumps in the dynamics of the patent value.

In spite of the relevance that jumps can take place in the real world, to the best of our knowledge, very little has been said in the literature, bacause most of the models are formalized in continuous time.

Within the real options approach we will present a very general model meant to provide a unifying theoretical framework where the existing models of patent valuation can be considered as special cases. The generality of the model also allows for unconsidered theoretical hypotheses, taking a step forward towards a more realistic model. As we will see, this can be achieved by incurring the cost of adopting an unusual process: the Spatial Mixed Poisson Process, SMPP.

Is this cost worth incurring? The answer must be given both in terms of theoretical advances the model can generate and in terms of economic implications.

As to the theoretical aspects, the approach can produce several new results with respect to the existing literature. First, it allows tackling the problem of the value of patents in the presence of both negative and positive jumps in the underlying. ${ }^{1}$ Second, the renewal process is not contemplated in that part of the literature which models (only negative) jumps through a Poisson process. ${ }^{2}$ At the same time, the renewal process has been extensively modeled in the literature, but dropouts have been modeled under the "no jumps" assumption. Our general model puts together the two strands of literature, modeling the jumping value of a patent in the presence of a renewal schedule. Specifically, the results generated by the model can be easily compared to those accruing from the studies related to the patent valuation with renewal schedule (from Pakes (1986) onward). In this respect, an explicit connection between real options and the patent renewal schedule literature is proposed. Third,

\footnotetext{
${ }^{1}$ As an example of positive jumps think of more effective or rigorous protection policies, such as the establishment of the Court of Appeals of the Federal Circuit by US Congress or the EU directive $2004 / 48$ on the enforcement of intellectual property rights.

${ }^{2}$ See for instance Weeds (2003), Lambrecht and Perraudin (2003), Schwartz (2004) and Miltersen and Schwartz (2004)
} 
we introduce the realistic feature that not any jump can kill the patent, but only a large enough jump can do it. This feature is consistent with the good news principle, as first spelled out by Bernanke (1983) and more recently taken up by Boyarchenko (2004) and the literature therein. The principle can be stated as follows. Suppose that a negative jump is followed by a positive one. If the firm disinvested, after the unlucky jump, but then the cash flow increased, the firm cannot invest again because of investment irreversibility. Hence, it was better not to disinvest. In our specific case disinvestment is represented by not paying the renewal fee, once the patentee misses one fee the patent cannot be resumed. Hence, the assumption that not any jump kills the patent is consistent with the good news principle. By the same token, the same argument applies for the bad news principle in case of investment decisions ${ }^{3}$.

Fourth, there is a random delay in the transmission of the jump from the underlying state to the patent value. Fifth, as the patent gets older, namely approaching the final date $\mathrm{T}$, its value becomes less sensitive to the jumps in the underlying asset. That is because the propagation can take place progressively rather than abruptly and also because some jumps in the underlying asset do not have enough time to propagate to the patent value. Therefore, we can realistically affirm that the sensitivity of the patent value decreases with respect to the time of the jumps. Sixth, the adoption of the SMPP avoids the unpleasant hypothesis of no expiration date (Schwartz and Moon (2000), Bloom and Van Reenen (2002)). Finally, the adoption of SMPP allows to take into account the dependence between time and size of jumps in the cash flow. We will turn to all these points in more details when introducing them formally.

These theoretical advances spawn relevant economic and policy implications. The main message from the model is that, in principle, the valuation can drastically differ and policy recommendations can be mistakenly inferred if one neglects the possibility of positive jumps, and the delay and decay in jumps propagation. Neither a sensible comparison of patent rights can be made between countries and sectors. Hence, if the value of patent rights is inadequately estimated, biased incentives will be fostered.

It is important to highlight that the cost to incur in terms of algebra to enjoy the benefits is not sunk, in the sense that the Mixed Poisson Processes on the line and the infinite-server queue models are widely known and used (see e.g. Grandell (1997)); and also a spatial setting can be useful in modeling several practical situations (e.g. spatial queues, see Cinlar (1995)).

From a purely technical point of view, the dynamics of the underlying state is assumed to evolve in discrete time and both sizes and times of the jumps are assumed to be stochastic variables. This set up allows to obtain an approximation formula of patent value by providing a computation of the total size of its jumps in a given period. Since patents are supposed to jump with a certain delay with respect to the occurrence of the event, the jumps that will occur "tomorrow" are partially due to the jumps in the economy that occur "today". Hence, to value a patent tomorrow one must have the knowledge of the jumps in the economy already occurred, the effect of which will be exerted tomorrow. Moreover, the information set to be used for the

\footnotetext{
${ }^{3}$ We are indebted with an anonymous referee for having raised this point.
} 
estimation contains also a sample of how external events impact on the underlying asset.

The remaining part of the paper is organized as follows. Section 2 briefly reviews the real options literature on patents valuation. Section 3 presents the set up of the model describing the dynamics of the underlying asset, constrained by some economic and statutory terms which patents are subject to. Section 4 presents the valuation mechanism for valuing a patent at a given point in time. Finally, Section 5 summarizes the results and provides some conclusive remarks. The main definitions and the key results on SMPPs are contained in the Appendix.

\section{Review of the literature}

The literature on patent valuation is very broad. In this short review we will restrict our attention to the contributions which tackle the problem with a real option perspective. Special attention will be paid to those contributions in which the underlying asset is supposed to undergo possible jumps.

As patents have the three characteristics of: i) partial irreversibility of the investment undertaken, ii) uncertainty of future payoffs, iii) possibility to delay the actions, a prominent part of the literature has regarded at the problem of patent valuation as one of the (real) options valuation. In this respect, some important papers are worth mentioning. Pakes (1986) deals with the analysis of future cash flow generated by a patent in presence of a renewal fee schedule, and solves the optimal stopping problem of the abandonment of a patented project. The main parameters of Pakes' model are estimable from aggregate data on proportions renewed and the author applies the model to some Western countries. The estimation of patents value in a renewal fee model is also faced by Pakes and Simpson (1989) who, differently from Pakes, adopt a parametric estimation strategy. Bloom and Van Reenen (2002) still formulate an estimable real option model, but taking advantage of citations data, rather than renewal, and estimate the impact of patents on firm-level productivity and market value. The analysis they propose is eminently empirical, as that of Laxam and Aggarwal (2003), where the value of a specific patent related to a telecommunication technology is assigned by means of the real option approach. Baudry and Dumont (2006) is a prominent Pakes (1986)'s follower. The authors regard at renewal schedules as a tool to evaluate patents in a more general context than that presented by Pakes and important policy implications are derived. Specifically, renewal fees can be used to discourage low-value patents, reducing the burden of patent offices. Indeed, the authors introduce a binomial tree framework, hence a discrete time framework in which the underlying asset takes on jumping values, to make their results comparable to a wide number of studies related to financial options.

In spite of the relevance that jumps can take place in the real world, to the best of our knowledge, very little has been said in the literature. Some exceptions are worth mentioning: Pennings and Lint (1997) is the first contribution dealing with patent evaluation in a real options framework in presence of jumps of stochastic amplitude obeying a Poisson law. The option value of the proposed model depends on the 
expected number and size of the jumps. The model is also verified with an empirical application to a company operating in the electronic field. We refer the reader to this paper for a review of earlier contributions on real options and jumps in other areas, different from patents. Schwartz (2004) models changes in regulatory environment as a possible jump in the Poisson process that the net cash flow generated by the project can undergo. In the spirit of McDonald and Siegel (1986) he considers only negative jumps affecting the value of the patent in two ways. First, reducing the expected rate of capital gain in the underlying asset, which reduces the value of the option. Second, increasing the variance of percentage changes in the underlying asset over finite time intervals, and this tends to increase the value of the option. The net effect is a reduction in the value of the option (patent). Pennings and Sereno (2011) are close to Schwartz (2004) for the application to pharmaceutical companies, and assume that the underlying asset is driven by a a mixed jump-diffusion law. The authors build a simple model, where the intervention of jumps is limited to the representation of the binary event of occurrence (or not) of technological failure. Also in this case, the arrival times are of Poisson-type.

In a context of strategic interactions, the real options approach has been extensively used (Hsu (2007) and Meng (2008) and the literature therein), and jumps in a Poisson process are used to capture other firms patent attainment (Miltersen and Schwartz (2004), Lambrecht and Perraudin (2003), Weeds (2002), Schwartz and Moon (2000)). According to a different approach, Baudry and Dumont (2006) give a definition of a very broad class of discrete time stochastic processes to describe the evolution of the rent generated by a patent. In such a way they model regulatory interventions, such as a change in the schedule of renewal fees, highlighting how the profile of renewal fees can reduce applications from worthless patents.

In keeping with the idea of Baudry and Dumont to use a new stochastic process, in the next section we will make use of the SMPP. To the best of our knowledge, few studies are present in the literature implementing SMPP processes in a real option context. The first contribution is due to Cerqueti et al. (2009) who develop a real option-like model with SMPP applied to insurance-reinsurance issues. Cerqueti (2013) proposes a similar setting to the problem of available stock of natural resources. Whereas, the contribution of Cerqueti and Ventura (2013) is closer to our paper, as they deal with a (would-be) patent related problem, still in the real option language with SMPP. Specifically, Cerqueti and Ventura (2013) model the $R \& D$ phase before patent attainment, and focus on competition and strategic interactions in a race context. In their work, the real option approach hinges on the optimal time for taking out a patent, as well as on discussing the payoff of the $R \& D$ phase. Differently, the model presented in the next section focuses on the valuation of a patent, right after the $R \& D$ phase.

\section{The model}

The model for the valuation of a patent presented hereafter is in accordance with the real options approach. The value of a patent, denoted by $C_{t}$, is supposed to be driven by the evolution of an underlying state. According to the literature, the underlying 
state is represented by the cash flow. We stress that the evolution of patent value can drastically change in the presence of impulsive events. A technological improvement or the introduction of laws modifying the protection rules can be reasons for a jump in the dynamics involved in the patent valuation process. Therefore, as already said in the Introduction, we rely on an underlying process able to provide a quantitative description of the qualitative shocks occurring in the economic environment, adopting a discrete time framework. To this respect, it is interesting to note that some models of patent valuation approximate the results obtained in continuous time with an approximate formulation in discrete time, recognizing the latter to be more realistic. "[...] The decision to abandon the project is evaluated at discrete points in time, instead of continuously. This would seem to be a more reasonable assumption when analyzing RED projects [...]" (Schwartz (2004) p. 52).

To make the model as clear as possible, we present it in a stepwise form.

\subsection{The cash flow components}

The cash flow is supposed to be composed by three parts: the "normal" component due to the net cash flow generated by the presence of the patented good on the market, plus the algebraic value of the jumps due to the events in the economy, minus the renewal fees. Substantially, for each time $t \geq 0$, we can write

$$
C_{t}=C_{t}^{N}+C_{t}^{J}-C_{t}^{F}
$$

where the superscripts $F, N$ and $J$ concern fees, normal flow and jumps, respectively.

To formalize the processes, let us consider a probability space with filtration $\left(\Omega, \mathcal{F},\left\{\mathcal{F}_{t}\right\}_{t \geq 0}, P\right)$ containing all the random variables used throughout the paper.

- The first component can be represented by a stochastic process $\left\{Y_{t}\right\}$, where $t$ takes values in a discrete time set $S=\left\{s_{k}\right\}_{k \in \mathbb{N}} \subseteq(0,+\infty)$, such that $Y_{t}$ are i.i.d., with mean $\mu$ and variance $\sigma^{2}$. To avoid unnecessary complexities of the model, we assume that the dates $s$ 's are deterministic, in that the net cash flows generated by the presence of the patented good in the market are measured at fixed times. However, our theoretical proposal remains valid also in the case of stochastic $s$ 's.

- To model the second component let us denote as $\mathcal{T}$ the set of the stopping times in $[0,+\infty)$, i.e.

$$
\mathcal{T}:=\left\{\tau: \Omega \rightarrow[0,+\infty) \mid\{\tau \leq t\} \in \mathcal{F}_{t}, \forall t \geq 0\right\}
$$

We introduce a stochastic point process $R=\left\{\left(\tau_{i}, \xi_{i}\right)\right\}_{i \in \mathbb{N}}$ capturing qualitative events and transforming them into quantitative amounts, where the coordinates $\tau_{i}$ and $\xi_{i}$ represent, respectively, the arrival time and the size of the $i$-th jump in the economy. The quantitative events impact on the cash flow through a transformed point process, $N$. The latter process represents the jumps in the cash 
flow generated by the jumps in $R$, namely the jumps in the underlying state generated by the events occurring in the economy. In particular, $\tau_{i} \in \mathcal{T}, i \in \mathbb{N}$, with $\tau_{i}$ taking values in $[0,+\infty)$ and $\xi_{i} \in \mathcal{F}_{\tau_{i}}, i \in \mathbb{N}$, with $\xi_{i}$ taking values in $\mathbb{R}$. From now on we will interchangeably refer to events or jumps, as they are two sides of the same coin, being the latter the quantitative image of the former. The random variables $\xi_{i}$ are i.i.d. and independent from the one-dimensional process $\left\{\tau_{i}\right\}_{i \in \mathbb{N}}$. It is worth noting that the events are random both in arrival time and in size.

In our setting we will consider $\xi_{i}>0$ as a good news for the patent value, and $\xi_{i}<0$ as a bad news. For instance, good news can be represented by any policy aimed at strengthening or widening the patentee's rights, such as a more efficient judicial system, a decrease in costs of suing an infringer, an enhancement of the enforcement system, a tougher infringer's punishment, an increase in the statutory limit, etc. Shortly, good news are such to produce additional opportunities to exploit the patented innovation, conversely for bad news (Baudry and Dumont (2006)).

Recall that the presence of jumps in the economy generates jumps in the underlying state, eventually causing the patent value to jump. We describe this mechanism by introducing a bivariate random variable $w_{i}=\left(w_{i}^{(1)}, w_{i}^{(2)}\right), i \in \mathbb{N}$, and a transformation $\phi$ as follows: $w_{i}$ takes values on $[0,+\infty)^{2} ; w_{i}^{(1)}$ and $w_{i}^{(2)}$ represent a stochastic delay and a stochastic scale factor, respectively, and they are assumed to be i.i.d.. The function $\phi$ is such that

$$
\phi:[0,+\infty) \times \mathbb{R} \times[0,+\infty)^{2} \rightarrow[0,+\infty) \times \mathbb{R},
$$

with

$$
\phi\left(\tau_{i}, \xi_{i}, w_{i}\right):=\left(\tau_{i}+w_{i}^{(1)}, w_{i}^{(2)} \xi_{i} \mathrm{e}^{-\tau_{i}}\right), \quad i \in \mathbb{N},
$$

The transformation $\phi$ explains how times and sizes of the jumps in the economy propagate to the cash flow of the patent. By defining

$$
\lambda_{i}:=\tau_{i}+w_{i}^{(1)}, \quad \gamma_{i}:=w_{i}^{(2)} \xi_{i} \mathrm{e}^{-\tau_{i}}, \quad \forall i \in \mathbb{N} .
$$

we can define the process $N$ of the jumps in the cash flow as a transformation of $R$ :

$$
N=\left\{\left(\lambda_{i}, \gamma_{i}\right)\right\}_{i \in \mathbb{N}}
$$

Remark 1. Even if the sizes of the stochastic jumps are theoretically unbounded, without loss of generality one can reasonably assume the existence of a positive upper and a negative lower threshold sufficiently large for the jumps sizes in the underlying state dynamics and patent value. Therefore, we assume hereafter the existence of two positive constants $a$ and $b$ such that, fixed $i \in \mathbb{N}, \xi_{i}$ and $\gamma_{i}$ are random variables with support in $[-a, a]$ and $[-b, b]$, respectively.

- The third cash flow component is a sequence of known positive amounts $\left\{X_{j}\right\}_{j \in \mathbb{N}}$ at deterministic fixed dates $T_{j}$, with $j \in \mathbb{N}$. 


\subsection{Economic interpretation of the process $N$}

The definition of $\phi$ is based on some important aspects allowing to make the valuation process as general as possible. We describe hereafter the benefits of the assumed functional form of $\phi$.

i) In accordance with the current literature, the presence of a jump in the underlying asset is meant to capture some stylized facts, for instance, when another firm takes out a patent on a new, more technologically-sophisticated product. The new product is supposed to make the valued patent immediately outdated. In the real world it takes time before the new product is marketed and, once marketed, it takes time before it is widely diffused to the point of cutting to zero the profits accruing from the old patent. According to the model, it takes time before a jump in the economy turns into a corresponding jump in the patent value. For instance, let's think of a new patent for some electronic device, such as a new cell phone or a new television incorporating technology improvements. It is quite evident that it takes time between the new patent registration and the complete obsolescence of the old patent. Especially for consumer electronics, elderly people are less willing to adopt new technologies, making still temporarily worth the old ones. In such a case, the assumption of a no-delay condition is a naïve approximation. At the same time, we do not deny that there can be circumstances in which the delay is almost null. For instance, it may be that clinical trials reveal that a patented drug has some terrible side effects, or it may be that the government prohibits certain classes of drugs (Miltersen and Schwartz (2004)). In order to make the transmission mechanism of the jump from the economy to the patent value as general as possible, we assume that there is a random delay between the time of occurrence of a shock in the economy and the time of the correspondent jump in the underlying state dynamics, i.e. in the patent value. The no delay case can thus be regarded as a special case, embedded into this very general assumption. This general transmission mechanism has practical spillovers when determining licensing or selling prices. If the parties do not take the transmission delay into account, the deal can finally set a too high (low) price whenever a negative (positive) jump in the economy occurs and is not yet propagated to the patent. At aggregate level, macro valuations of patents rights can end up with over/under estimates. It follows that if important patent-protection measures, concerning other means of returns-appropriation from invention, are constructed ${ }^{4}$, misleading policy implications can be drawn and misleading comparison can be made between countries and sectors.

ii) The value of a patent falls (rises) when a bad (good) news appears in the economic system, i.e. the presence of a jump of negative (positive) size. The entity of the rise or fall of the patent value is not fully controlled by the economic actors, i.e., the patentees.

\footnotetext{
${ }^{4}$ Such as the Equivalent Subsidy Rate, ESR, given by the ratio of the total value of patent rights relative to $R \& D$ used to produce those patents.
} 
iii) The value of the patent is increasing with respect to the size of the jumps in the underlying asset.

iv) The propagation process can be progressive rather than abrupt and some jumps in the economy have not enough time to propagate to the patent value. Therefore, when modeling the realistic feature, we also consider that the sensitivity of the patent value decreases with respect to the time of the jumps. Formally, if two jumps of the same size occur at two different points in time, say $\tau_{1}<\tau_{2}$ the value of the patent has a less remarkable jump in $\tau_{2}$, since the end of the patent life is nearer. As a by-product, this sort of decay in the strength of the jumps, associated to the delay in the transmission mechanism, allows to capture those limiting cases in which the jump in the economy is not transmitted to the patent because too close to expiry. For instance, suppose a rival firm takes out a new patent when the old one is close to expiration. The expected profit loss will be almost negligible, both because of the residual short life of the patent and because of the delay in the jump propagation to the patent value.

Delay and decay, as explained in points i) and iv), confer the patent valuation approach the realistic feature to be able to generate worthless patents before the statutory term, even without the occurrence of a catastrophic event. Indeed, some jumps occurring in the economy in a final part of the patent life can be not transmitted to the patent and those jumps transmitted may have a little impact. Indeed, as the patent ages there are few years in which the patent can earn returns, renewal fees rise making the patent to be worthless. This feature is not accounted for in the other models, which necessarily require a killing jump to put an end to the patent life. In this respect, the model is consistent with the empirical finding by Shankerman (1998), Lanjouw et al (1998) and Pakes and Simpson (1989) who report that only a negligible part of patents reaches the final statutory term.

\subsection{The null patent conditions}

In the spirit of Pindyck (1993), in our context the process $R$ affects $N$ that drives the value of the patent. Hence, as the patent valuation theory states, the value of a patent is null when a negative jump with a large enough size occurs. Define as $\tau^{\star} \in \mathcal{T}$ the random time at which a negative jump with a large enough size occurs. Then one of the following alternatives holds:

- $\tau^{\star}=+\infty$;

$\bullet \exists j \in \mathbb{N} \mid \tau^{\star} \equiv \tau_{j}$

Moreover, patents are subject to a statutory limit, i.e. the expiration date $T>0$, when the patent loses its value and the production of the protected good becomes of public domain ${ }^{5}$. Hence, the underlying state must be observed till the threshold $T$ if

\footnotetext{
${ }^{5}$ Generally, $T=20$ years.
} 
a negative jump large enough does not occur before.

Importantly, a delay is assumed between jumps in the economy and jumps in the cash flow, but no delay occurs between jumps in the cash flow and jumps in the patent value. Put another way, differently from the relationship between economic environment and cash flow, the jumps in the cash flow propagate immediately on the value of the patent, without any delay. Patents owe their value to the cash flow they can generate. It follows that one must rule out situations wherein patents take on values different from zero, although they are unable to generate positive cash flow. That would happen if a delay occurred between a negative large enough jump in the cash flow and the corresponding jump in the patent.

Denote as $\lambda^{\star}$ the delay of the stopping time $\tau^{\star}$, according to formula (4). Consistently with a deadly jump in $R$ at $\tau^{\star} \phi$ propagates that jump to the patent at the random time $\lambda^{\star} \geq \tau^{\star}$.

Hence, the entire cash flow sequence stops naturally in three situations:

- when the expiration date $T$ is reached;

- after the random time $\lambda^{\star}$, e.g. when the value of the underlying asset becomes null. In this case, obviously, the last periodical payment is the one immediately before the stopping time $\lambda^{\star}$;

- if the patentee reckons that patent renewal is not a suitable economic strategy. This can happen if the expected net gain from holding the patent is negative. Formally, for the time being, we can assume that there exists a deterministic time-varying threshold $\Psi(t)$ such that $X_{k}=0$, for each integer $k>j^{\star}$, where

$$
j^{\star}:=\min \left\{j \in \mathbb{N} \mid X_{j} \geq \Psi\left(T_{j}\right)\right\} .
$$

We will refer to $\Psi(t)$ as the renewal threshold.

Remark 2. In the patent value literature, some studies focus on the construction of endogenous models for $\Psi(t)$ and derive the optimal stopping related to the renewal process. In this respect, the Bellman principle has been used both in deterministic and stochastic framework (see Pakes (1986) and Pakes and Shankerman (1986)). Of course our model can be re-formulated in terms of the Bellman equation, but this is far beyond the scope of the paper and it would imply the writing of a new paper. We leave this intriguing issue to future research. Rather than an optimization model, we deal with the construction of patent value dynamics in a very general stochastic set up, characterized by unpredictable jumps, in order to concentrate on the effects of the jumps on the patent value when allowing for delay and decay phenomena.

In order to formalize the intervention of the critical index $j^{\star}$ we define the timedependent property $\Pi_{t}$ as

$$
\Pi_{t}:=\left\{T_{j^{\star}} \leq t\right\} .
$$

indicating the renewal time at which the patentee decides not to renew the patent. When the patent is not renewed, its future cash flow obviously becomes null from 
that time onward. More specifically, when the underlying asset dynamics reaches the expiration date $T$ or the patent is not renewed, the cash flow becomes immediately null, without time transformations. Differently, when the economy undergoes a large enough jump the cash flow becomes null at the random time-transformation of $\tau^{\star}$, i.e. in $\lambda^{\star}$ and the patent immediately dies.

\subsection{The patent value}

We introduce an appropriate discount factor $\beta \in(0,1)$ and denote the patent value at time $t$ as $C_{t}$. As clearly explained by Pakes and Simpson (1989) and Shankerman and Pakes (1986), $C_{t}$ can be regarded as the value of patent protection to the patentee before the next patent renewal. Actually, $C_{t}$ is a random variable in that it is given by the future random cash flow. As we will see later, an estimation of the patent value will be obtained through the computation of its conditioned expected value.

If the renewal fee is paid, the patentee earns the current return to patent protection, provided that a deadly jump has not intervened yet. Measuring the value of patent protection by the net present value, the value of the patent equals the future expected discounted value of the cash flow from holding the patent.

To fix notation, we distinguish two cases.

- If $t<\lambda^{\star} \wedge T$ and property $\Pi_{t}$ does not hold, then

$$
C_{t}=\sum_{i=1}^{+\infty} \gamma_{i} \beta^{\lambda_{i}-t} \mathbf{1}_{\left\{t \leq \lambda_{i} \leq \lambda^{\star} \wedge T\right\}}-\sum_{j=1}^{+\infty} X_{j} \beta^{T_{j}-t} \mathbf{1}_{\left\{t \leq T_{j} \leq \lambda^{\star} \wedge T\right\}}+\sum_{k=1}^{+\infty} Y_{k} \beta^{s_{k}-t} \mathbf{1}_{\left\{t \leq s_{k} \leq \lambda^{\star} \wedge T\right\}}
$$

The value of a patent at time $t$, when a large enough jump in the underlying asset has not occurred, $t<\lambda^{\star}$, and the final expiration date, $T$, has not been reached is given by the expected discounted sum of the three parts of the cash flow. The discounted value of the jumps, minus the term related to all the discounted renewal fees, plus the future expected normal cash flow.

- Otherwise

$$
C_{t}=0
$$

\section{Estimation of the patent value}

This section is devoted to provide a mechanism for the computation of the patent value at a given time $t$.

First of all, a discussion on the randomness of the process involved in the patent value is needed.

There is a clear correlation between $\gamma_{i}$ and the $\left(\tau_{i}, \xi_{i}\right)$ couple. This fact does not allow to consider $R$ as a stochastic process on the line, and we need to treat $R$ as a Spatial Point Process. The following Assumption will stand in force hereafter:

Assumption 1. $R$ is a $S M P P$. 
For the formal definition of SMPP, see Definition 1 in the Appendix.

The statement of Assumption 1 is important for two reasons. First of all, it contributes to construct a model for simultaneously computing the number and the size of the jumps in the economy and, consequently, the number and the size of the jumps in the patent value dynamics. Secondly, according to some recent results, SMPPs can guarantee the invariance of the stochastic structure between $R$ and $N$. More precisely, if $R$ is a SMPP, then the process $N$ is a SMPP too (see the Appendix). This is a key result for the estimation procedure.

In order to proceed, we now need to define a time interval

$$
\mathcal{I}:=\left[0, T^{\prime}\right]
$$

with $T^{\prime} \leq T$. The interval $\mathcal{I}$ represents the period under scrutiny, and it plays a key role in providing the estimate we are dealing with.

We now define the regions $I \equiv \mathcal{I} \times[-a, a]$ and $J \equiv \mathcal{I} \times[-b, b]$. Moreover, in agreement with a common used mathematical notation, we introduce $\triangle \subseteq \mathbb{R}^{2}$ and denote by $R(\triangle)$ and $N(\triangle)$ the number of the elements of the Spatial Point Process $R$ and $N$ respectively, that are contained in $\triangle$. Further, let us define the random subset of indexes $\left\{i_{1}, \ldots, i_{K}\right\} \subset \mathbb{N}$, such that

$$
\left\{\left(\tau_{i_{1}}, \xi_{i_{1}}\right), \ldots,\left(\tau_{i_{K}}, \xi_{i_{K}}\right)\right\}=I \cap R .
$$

We notice that $K \equiv R(I)$.

The stopping time $\tau^{\star}$ is located at random jumping time $\tau_{i_{\bar{K}}}$, where $\bar{K} \in \mathbb{N}$ or, alternatively, $\tau^{\star}=+\infty$. We denote by $I_{\tau^{\star}}$ the restriction of the counting spatial process $R$ in the set $I$ up to the stochastic threshold $\tau^{\star}$. Formally, we have

$$
\left\{\left(\tau_{i_{1}}, \xi_{i_{1}}\right), \ldots,\left(\tau_{i_{n \wedge \bar{K}}}, \xi_{i_{n \wedge \bar{K}}}\right)\right\}_{n=1 \ldots, K}=I_{\tau^{\star}} \cap R
$$

and $K \wedge \bar{K} \equiv R\left(I_{\tau^{\star}}\right)$.

Equally, let us denote the random subset of indexes $\left\{i_{1}, \ldots, i_{\tilde{K}}\right\} \subset \mathbb{N}$, such that

$$
\left\{\left(\lambda_{i_{1}}, \gamma_{i_{1}}\right), \ldots,\left(\lambda_{i_{\tilde{K}}}, \gamma_{i_{\tilde{K}}}\right)\right\}=J_{\tau^{\star}} \cap N
$$

where $J_{\tau^{\star}}$ denotes the propagation on the process $N$ in the set $J$ of the restriction of $R$ due to the presence of the stopping time $\tau^{\star}$. We notice that $\tilde{K} \equiv N\left(J_{\tau^{\star}}\right)$.

Furthermore, property $\Pi_{t}$ implies the existence of an index $\tilde{K}^{\star} \leq \tilde{K}$ such that $\tilde{K}^{\star}<$ $j^{\star} \leq \tilde{K}^{\star}+1$. We denote as $J_{\tau^{\star}, \star}$ the further restriction on $J_{\tau^{\star}}$ driven by property $\Pi_{t}$. We have

$$
\left\{\left(\lambda_{i_{1}}, \gamma_{i_{1}}\right), \ldots,\left(\lambda_{i_{\tilde{K}^{\star}}}, \gamma_{i_{\tilde{K}^{\star}}}\right)\right\}=J_{\tau^{\star}, \star} \cap N .
$$

We also consider the random subset of indexes $\left\{i_{1}^{\prime}, \ldots, i_{K^{\prime}}^{\prime}\right\} \subseteq\left\{i_{1}, \ldots, i_{n \wedge \bar{K}}\right\}_{n=1, \ldots, K}$ such that the delay $\lambda_{i}$, with $i \in\left\{i_{1}^{\prime}, \ldots, i_{K^{\prime}}^{\prime}\right\}$, falls in the set $J$. We have a conditioning on the set $I_{\tau^{\star}}$. Taking into account property $\Pi_{t}$, we finally define:

$$
\left\{\left(\lambda_{i_{1}^{\prime}}, \gamma_{i_{1}^{\prime}}\right), \ldots,\left(\lambda_{i_{\tilde{K}}^{\prime}}, \gamma_{i_{\tilde{K}}^{\prime}}\right)\right\} \cap\left\{\left(\lambda_{i_{1}}, \gamma_{i_{1}}\right), \ldots,\left(\lambda_{i_{\tilde{K}^{\star}}}, \gamma_{i_{\tilde{K}^{\star}}}\right)\right\}=J_{\tau^{\star}, \star} \cap N \mid I_{\tau^{\star}} .
$$

We denote $K^{\prime} \wedge \tilde{K}^{\star} \equiv N_{\left(I_{\tau^{\star}}\right)}\left(J_{\tau^{\star}, \star}\right)$. Evidently, $P\left(N_{\left(I_{\tau^{\star}}\right)}\left(J_{\tau^{\star}, \star}\right) \leq R\left(I_{\tau^{\star}}\right)\right)=1$. 
Remark 3. Since a SMPP is a simple process (see Appendix, Lemma 1) and regions $I_{\tau^{\star}}$ and $J_{\tau^{\star}, \star}$ are bounded, then $\mathbb{E}\left[R\left(I_{\tau^{\star}}\right)\right]<+\infty$ and $\mathbb{E}\left[N\left(J_{\tau^{\star}, \star}\right)\right]<+\infty$.

We now apply the approach of Cerqueti et al. (2009) to provide an approximation of the expected value of the patent at time $t$ in formula (7). The mechanism is based on the knowledge of the both the jumps in the economy and in the cash flow occurring over the time interval $\mathcal{I}$. Define the time interval $H=\left[T^{\prime}, T\right]$, the region $\mathcal{H}=H \times[-b, b]$ and also consider $t \in H$.

Let $\Upsilon=\left\{j_{1}, \ldots, j_{K^{\prime \prime}}\right\}$ be the random subset of indexes such that $\left\{\left(\lambda_{j_{1}}, \gamma_{j_{1}}\right), \ldots,\left(\lambda_{j_{K^{\prime \prime}}}, \gamma_{j_{K^{\prime \prime}}}\right)\right\}=$ $H_{\tau^{\star}, \star} \cap N$. As usual, $H_{\tau^{\star}, \star}$ denotes the restrictions on the process $N$ in $H$ due to the presence of the threshold $\tau^{\star}$ for $R$ and the property $\Pi_{t}$ for $N$. By definition of the process $N$, we can also write $K^{\prime \prime} \equiv N\left(H_{\tau^{\star}, \star}\right)$.

We omit hereafter the subscripts $\tau^{\star}$ in $I$ and $\tau^{\star}, \star$ in $J$ and $H$ to have a less cumbersome notation. Nevertheless, the computed quantities have to be intended under the threshold condition $\tau^{\star}$ for the process $R$ and under the property $\Pi_{t}$ for the process $N$. In this framework, the random amount $C_{t}^{J}$ can be written as follows:

$$
C_{t}^{J} \equiv \sum_{j \in \Upsilon} \gamma_{j}
$$

We will approximate the conditional expectation of $C_{t}^{J}$ given:

- the number $R(I)$ of jumps in the economy during the interval $\mathcal{I}$;

- the number $N(J)$ of the jumps in the cash flow, and therefore in the patent during $\mathcal{I}$;

- the number of jumps in the economy occurred over $\mathcal{I}$ propagated to the patent in the same time interval. We denote this quantity as $N_{(I)}(J)$.

We consider two partitions $\Delta$ and $\Psi$ of $H$ as follows:

$$
\Delta_{k}:=\left\{H_{s}^{(k)}\right\}_{s=1, \ldots, k}, \quad k \in \mathbb{N},
$$

where $H_{s}^{(k)}:=H \times\left(c_{s-1}^{(k)}, c_{s}^{(k)}\right]$, with $s=1, \ldots, k, c_{0}^{(k)}=-b, c_{k}^{(k)}=b$ and, for each $k$, $\left\{c_{s}^{(k)}\right\}$ is increasing with respect to $s$;

$$
\Psi_{h}:=\left\{G_{v}^{(h)}\right\}_{v=1, \ldots, h}, \quad h \in \mathbb{N},
$$

where $G_{v}^{(h)}:=\left(t_{v-1}^{(h)}, t_{v}^{(h)}\right] \times[-b, b]$, with $v=1, \ldots, h, t_{0}^{(h)}=T^{\prime}, t_{h}^{(h)}=T$ and, for each $h,\left\{t_{v}^{(h)}\right\}$ is increasing with respect to $v$.

A further refined partition of $H$ can be obtained by the intersection of the partitions defined in (10) and (11). We have

$$
\Delta_{k} \cap \Psi_{h}=\left\{H_{s}^{(k)} \cap G_{v}^{(h)}\right\}_{v \leq h ; s \leq k} \quad k, h \in \mathbb{N},
$$


Fix the four integers $s, v, h, k$. We denote by $b_{s, v}^{(k, h)}$ the expected number patent jumps observed in the time interval $\left(t_{v-1}^{(h)}, t_{v}^{(h)}\right]$ with size $\left(c_{s-1}^{(k)}, c_{s}^{(k)}\right]$, conditioned on the previous history in period $\mathcal{I}$, i.e.

$$
b_{s, v}^{(k, h)} \equiv \mathbb{E}\left[N\left(H_{s}^{(k)} \cap G_{v}^{(h)}\right) \mid R(I)=n^{\prime}, N(J)=n^{\prime \prime}, N_{(I)}(J)=m\right] .
$$

The next result provides a closed form expression to compute $b_{s, v}^{(k, h)}$, for any $(k, h) \in \mathbb{N}^{2}$ and $(s, v) \in\{1, \ldots, k\} \times\{1, \ldots, h\}$. Evidence is given in Cerqueti et al. (2009).

\section{Proposition 1.}

$$
\begin{gathered}
b_{s, v}^{(k, h)}=\sum_{n=0}^{+\infty} \sum_{l=0}^{n} n \frac{\left[M_{(\bar{I})}^{\star}\left(H_{s}^{(k)} \cap G_{v}^{(h)}\right)\right]^{n-l}}{(n-l) !} \frac{\left[M_{(I)}^{\star}\left(H_{s}^{(k)} \cap G_{v}^{(h)}\right)\right]^{l}}{l !} \times \\
\times \int_{0}^{+\infty} \lambda^{n-l} e^{-\lambda M_{(\bar{I})}^{\star}\left(H_{s}^{(k)} \cap G_{v}^{(h)}\right)} u\left(\lambda ; I, J, n^{\prime}, n^{\prime \prime}, m\right) \mathrm{d} \lambda \cdot \int_{0}^{+\infty} \lambda^{l} e^{-\lambda M_{(I)}^{\star}\left(H_{s}^{(k)} \cap G_{v}^{(h)}\right)} u\left(\lambda ; I, J, n^{\prime}, n^{\prime \prime}, m\right) \mathrm{d} \lambda,
\end{gathered}
$$

where

$$
u\left(\lambda ; I, J, n^{\prime}, n^{\prime \prime}, m\right)=\frac{\lambda^{n^{\prime \prime}-m+n^{\prime}} e^{-\lambda\left[M_{(\bar{I})}^{\star}(J)+M(I)\right]} u(\lambda)}{\int_{0}^{\infty} \lambda^{n^{\prime \prime}-m+n^{\prime}} e^{-\lambda\left[M_{(\bar{I})}^{\star}(J)+M(I)\right]} u(\lambda) d \lambda}
$$

is the posterior distribution on $\Lambda$ defined in Eq. (23).

Proposition 1 allows to get an upper and a lower approximation of $\mathbb{E}\left[C_{t}^{J} \mid R(I)=\right.$ $\left.n^{\prime}, N(J)=n^{\prime \prime}, N_{(I)}(J)=m\right]$.

Consider the following sequences:

$$
\begin{aligned}
& \bar{\phi}_{k, h}:=\sum_{s=1}^{k} \sum_{v=1}^{h} c_{s}^{(k)} \beta^{-\left(t-t_{v-1}^{(h)}\right)} b_{s, v}^{(k, h)}, \\
& \hat{\phi}_{k, h}:=\sum_{s=1}^{k} \sum_{v=1}^{h} c_{s-1}^{(k)} \beta^{-\left(t-t_{v}^{(h)}\right)} b_{s, v}^{(k, h)} .
\end{aligned}
$$

For any $h, k \in \mathbb{N}$, we have

$$
\hat{\phi}_{k, h} \leq \mathbb{E}\left[C_{t}^{J} \mid R(I)=n^{\prime}, N(J)=n^{\prime \prime}, N_{(I)}(J)=m\right] \leq \bar{\phi}_{k, h} .
$$

$\left\{\hat{\phi}_{k, h}\right\}$ is non-decreasing and $\left\{\bar{\phi}_{k, h}\right\}$ is non-increasing with respect to $k$ and $h$. Moreover, there exists a nonnegative constant dependent on $t$, named $p(t)$, such that

$$
\lim _{k, h \rightarrow+\infty} \hat{\phi}_{k, h}=\lim _{k, h \rightarrow+\infty} \bar{\phi}_{k, h}=p(t) .
$$

By (14), taking the limit, we can conclude that

$$
\mathbb{E}\left[C_{t}^{J} \mid R(I)=n^{\prime}, N(J)=n^{\prime \prime}, N_{(I)}(J)=m\right]=p(t) .
$$


Assume that $F_{\lambda^{\star}}$ is the (marginal) distribution function of $\lambda^{\star}$.

We have

$$
\begin{gathered}
\mathbb{E}\left[C_{t} \mid R(I)=n^{\prime}, N(J)=n^{\prime \prime}, N_{(I)}(J)=m\right]= \\
=p(t)-\mathbb{E}\left[\sum_{j=1}^{+\infty} X_{j} \beta^{T_{j}-t} \cdot \mathbf{1}_{\left\{T_{j} \in\left[t, \lambda^{\star} \wedge T\right]\right\}}\right]+\mathbb{E}\left[\sum_{k=1}^{+\infty} Y_{k} \beta^{s_{k}-t} \mathbf{1}_{\left\{s_{k} \in\left[t, \lambda^{\star} \wedge T\right]\right\}}\right]= \\
=p(t)-\sum_{j=1}^{+\infty} X_{j} \beta^{T_{j}-t} \cdot \mathbf{1}_{\left\{T_{j} \in[t, T]\right\}} \cdot P\left(T_{j} \leq \lambda^{\star}\right)+\sum_{k=1}^{+\infty} \mu \beta^{s_{k}-t} \cdot \mathbf{1}_{\left\{s_{k} \in[t, T]\right\}} \cdot P\left(s_{k} \leq \lambda^{\star}\right)= \\
=p(t)-\sum_{j=1}^{+\infty} X_{j} \beta^{T_{j}-t} \cdot \mathbf{1}_{\left\{T_{j} \in[t, T]\right\}} \cdot\left[1-F_{\lambda^{\star}}\left(T_{j}\right)\right]+\sum_{k=1}^{+\infty} \mu \beta^{s_{k}-t} \cdot \mathbf{1}_{\left\{s_{k} \in[t, T]\right\}} \cdot\left[1-F_{\lambda^{\star}}\left(s_{k}\right)\right] . \quad
\end{gathered}
$$

\subsection{Economic discussion of the mathematical result}

Equation (16) provides the value of the patent at time $t$. This value is different from zero under the constraints relative to the negative jump large enough, the renewal threshold and the expiration date.

It is worth noting that Equation (16) is perfectly in line with the literature on renewals (e.g. Shankerman and Pakes (1986) eq. (1), Pakes and Simpson (1989) eq. (1), Baudry and Dumont (2006) eq. (4)), in that the value of the patent at time $t$ is nothing but the expected net gain discounted from the valuation time, $t$, till its possible death. Not surprisingly, this value is given by the sum of three components. A first component stemming from the discounted expected value of the jumps in the underlying asset, $p(t)$; a second negative component due to the discounted value of the renewal fees payable in the future. In this quantity, the term $\left[1-F_{\lambda^{\star}}(\cdot)\right]$ captures the presence of the stopping time $\lambda^{\star}$ associated to a "catastrophic jump" of the underlying asset, responsible for the collapse of the patent value. That is, if a renewal fee $X_{j}$ is due in a time $T_{j}$ smaller than the delay $\lambda^{\star}$ of the stochastic time $\tau^{\star}$, it has to be inserted in the computation. Otherwise $X_{j}$ does not feed into the summation in (16). Finally, a third component concerning the expected discounted value of the normal flow, where the same agument about $\left[1-F_{\lambda^{\star}}(\cdot)\right]$ applies.

Despite its intuitive significance and the close similarity to the aforementioned literature, (16) reveals some critical considerations. Recall that $p(t)$ is a theoretical computation of the aggregate discounted cash flow at a given time $t$ due to the jumps in the economy, conditional on past information. Looking at the time profile of the computation of patent value, we can claim that $p(t)$ comes form the the algebraic sum of three components:

$$
p(t)=p_{H}^{(a)}(t)+p_{H}^{(b)}(t)-p_{H}^{(c)}(t)
$$

where $p_{H}^{(a)}(t)$ represents the jumps in the patent due to the jumps in the economy occurred in $H ; p_{H}^{(b)}(t)$ represents the jumps in the patent value due to the jumps in the economy occurred before $H$ and propagated in $H$; finally, $p_{H}^{(c)}(t)$ represents the jumps in the patent due to the jumps in the economy occurred in $H$ and propagated 
after $H$.

For given renewal schedule and normal flow, formula (17) is useful to compare the patent value obtained under (16) with the patent value obtained under an alternative naive model. By naive model, we mean a model in which no delay between the economy and the patent jump is assumed. Under this restrictive hypothesis, let us denote by $\tilde{p}_{H}(t)$ the aggregation of the expected discounted value of the jumps in the cash flow at time $t$, due to the jumps in the economy over a time interval $H$. The no delay condition implies that

$$
\tilde{p}_{H}(t)=p_{H}^{(a)}(t)
$$

where $p_{H}^{(a)}(t)$ is defined as in $(17)$.

By $(17)$ and (18), we generally have that $p(t) \neq \tilde{p}_{H}(t)$, since there is no reason to expect $p_{H}^{(b)}(t)=p_{H}^{(c)}(t)$. Therefore, we argue that paying a little price in terms of algebraic effort, the model better fit the real world, providing us with the patent value in a closed form solution. More remarkably, (16) and (17) show potential source of bias when estimating the value of patents in the "no delay" hypothesis. So far, the comparison between our model and an alternative naive one has been kept as simple as possible, but the bias can be worsened if one considers also that naive models contemplate neither the possibility of positive jumps nor the decay process of the transmission mechanism. Furthermore, if a naive model is applied to determine the value of patent rights in two countries or in two sectors, there is no reason to believe that the two biases will take on the same sign and the same intensity, hence the comparison can be meaningless. In other words, $p(t)=\tilde{p}_{H}(t)$ is a special case of (16).

A quantitative appraisal of the difference is far beyond the scope of this paper. We limit ourselves to bring it to notice.

\section{Conclusions and further research}

This paper is a generalization of the existing models for valuing a patent in a real options framework. Briefly speaking, the generalization consists in accounting for positive and negative random jumps, inducing possible delays in the transmission mechanism between the jumps in the underlying process and the corresponding jumps in the patent value, considering a decay process of the intensity of the shocks occurred to the economy and considering also the dependence between size and time of the jumps in the cash flow due to jumps in the economy. In this respect, if a shock intervenes in the economy when the patent is close to expiration, caeteris paribus, it will affect the patent with lower intensity.

We have suggested that the inclusion of these hypotheses is essential to add realism to the valuation process. In turn, this step also brings significant improvements, such as a solution to patent valuation in a closed form, without assuming the absence of expiration and considering the presence of non killing jumps. It follows that patent evaluation can greatly differ whether our model is adopted or a more naive approach is followed. Many factors account for that difference. Empirical works (Schankerman 
(1998), Lanjouw et al. (1998)) report that the distribution of the value of patents is highly skewed, there are many patents with low value and just a few with a high one. Our model sensibly assumes that when a low-valued patent is taken out by a competing firm, the high-valued patents undergo a non deadly jump. Therefore, the number of these jumps is quite far from being negligible and, consequently, the cumulated change in the high-valued patent is not marginal too. Moreover, the delay in the transmission mechanism is such that the patents value is accidentally equal under the two models, and the differences in both sign and magnitude can be hardly predicted.

Giving an estimate of the value of patent rights through a naive model, from a policy point of view, can lead to biased results that hinder a meaningful comparison between sectors and countries. If policy recommendations and interventions are based on a biased comparison, misleading incentives may be put into play.

From a theoretical point of view, it is worth stressing that the dependence of the $\gamma$ 's on the $\tau$ 's prevents to deal with processes on the line and motivates the assumption that $R$ is a SMPP. In fact, consider the familiar simple model described as follows: (i) the process $\left\{\tau_{i}\right\}_{i \in \mathbb{N}}$ is a one-dimensional Mixed Poisson Process; (ii) the $\xi$ 's are i.i.d. and independent of the $\tau$ 's; (iii) the $\gamma^{\prime}$ s are i.i.d. and independent from the process $R$.

Then, $N$ has the same stochastic structure described by the process $R$ identified by conditions $(i)$ and $(i i)$. In our specific case, as condition (iii) does not hold, $R$ and $N$ do not have the same structure. To state an invariant property between $R$ and $N$ and, at the same time, maintaining the validity of conditions $(i)-(i i i)$, we need to abandon the usual Mixed Poisson framework and let the process $R$ be a SMPP. This allows us to maintain the model as general al possible and to proceed with the estimation.

Another key point that would be worthwhile tackling in a succeeding study is the empirical evaluation of patent rights under the present approach, through an econometric estimate. A natural comparison between our model and Pennings and Lint (1997) can be then put forward: indeed, Pennings and Lint (1997) model can be viewed as a benchmark model, and our framework as an extension. Specifically, in Pennings and Lint (1997) the cash flow process does not include delays and timevariations of the jump times. However, Pennings and Lint do not deal with SMPPs, and so their paper is radically different from the present one. Having said this, it is important to note that simulating a SMPP represents a challenging issue, which has never been treated in the literature. We are currently working in this direction.

\section{References}

[1] Baudry M., Dumont B., 2006. Patent Renewals as Options: Improving the Mechanism for Weeding Out Lousy Patents. Review of Industrial Organization, 28, 41-62. 
[2] Bernanke B.S., 1983. Irreversibility, Uncertainty and Cyclical Investment. Quarterly Journal of Economics, 98(1), 85-106.

[3] Bloom N., Van Reenen J., 2002. Patents, Real Options and Firm Performance, The Economic Journal, 112(478), C97-C116.

[4] Boyarchenko S., 2004. Irreversible Decisions and Record-Setting News Principle. American Economic Review, 94(3), 557-568.

[5] Cerqueti, R., 2013. Exhaustion of resources: a marked temporal process framework. Stochastic Environmental Research and Risk Assessment, doi:10.1007/s00477-013-0798-7.

[6] Cerqueti, R., Foschi, R., Spizzichino, F., 2009. A Spatial Mixed Poisson Framework for Combination of Excess-of-Loss and Proportional Reinsurance Contracts. Insurance: Mathematics and Economics, 45(1), 59-64.

[7] Cerqueti, R., Ventura, M., 2013. Risk and uncertainty in the patent race: a probabilistic model. IMA Journal of Management Mathematics, doi:10.1093/imaman/dpt020.

[8] Chang, T.C.E., Sim, L.S.M., 1993. The Assessment of Reservation Prices of an Intermediate $R \& D$ Result and Patent Reward Under Oligopolistic Competitive Behaviour. Journal of the Operational Research Society, 44, 1225-1233.

[9] Cinlar, E., 1995. An introduction to spatial queues. In: Advances in Queueing, Probability and Stochastics Series, CRC, Boca Raton, 103-118.

[10] Crump, K., 1975. On point processes having an order statistic structure. Sankhya Ser. A, 37, 396-404.

[11] Daley, D.J., Vere-Jones, D., 1988. An Introduction to the Theory of Point Processes. New York: Springer-Verlag.

[12] Dixit A. and Pyndick R., 1994. Investment Under Uncertainty, Princeton University Press.

[13] Foschi, R., Spizzichino, F., 2008. The role of the Order Statistic Property in Mixed Spatial Poisson Processes. In: Proceedings of the International Workshop on Applied Probability. Université de Technologie de Compiègne.

[14] Grandell, J., 1997. Mixed Poisson Processes. Chapman \& Hall, London.

[15] Hsu, Y., and B.M. Lambrecht, 2007. Preemptive Patenting under Uncertainty and Asymmetric Information. Annals of Operations Research, 151 (1), 5-28.

[16] Laxman P.R., Aggarwal, S., 2003. Patent valuation using real options, IIMB Management Review, 15(4), 44-51. 
[17] Lambrecht B., Perraudin W., 2003. Real Options and Preemption under incomplete information. Journal of Economic Dynamics and Control, 27(4), 619-643

[18] Lanjouw J.O., Pakes A., Putnam J. 1998. How to Count Patents and Value Intellectual Property: the Uses of Patent Renewal and Application Data", The Journal of Industrial Economics, 46(4), 405-432

[19] Meng, R., 2008 A patent race in a real options setting: Investment strategy, valuation, CAPM beta, and return volatility. Journal of Economic Dynamics and Control 32 (10), 3192-3217.

[20] Miltersen K.R., Schwartz E.S., 2004. R\&D Investments with Competitive Interactions, Review of Finance, 8(3), 355-401

[21] McDonald R., Siegel D., 1986. The Value of Waiting to Invest. Quarterly Journal of Economics, 101 November, 707-728.

[22] Mirasol, N.M., 1963. The output of an M/G/ $\infty$ queueing system is Poisson. Operations Research 11, 282-284.

[23] Pakes, A.S., 1986. Patents as Options: some Estimates of the Value of Holding European Patent Stocks. Econometrica 54(4), 755-784.

[24] Pakes A.S., Simpson M., 1989. Patent Renewal Data, Brooking Papers on Economic Activity: Microeconomics, 331-410

[25] Pennings E., Lint O., 1997. The Option value of Advanced R\&D, European Journal of Operational Research, 103(3), 83-94.

[26] Pennings E., Sereno L., 2011. Evaluating Pharmaceutical R\&D Under technical and Economic Uncertainty, European Journal of Operational Research, 212, 374385.

[27] Pindyck R.S., 1993. Investments of uncertain cost. Journal of Financial Economics, 34, 53-76

[28] Renyi, A., 1967. Remarks on the Poisson process. Studia Scientiarum Mathematicarum Hungarica, 2, 119-123.

[29] Ross, S.M. 1996. Stochastic processes. New York: John Wiley.

[30] Schankerman M, 1998. How Valuable is Patent Protection? Estimates by Technology Field, Rand Journal of Economics, 29(1), 77-107.

[31] Schankerman M., Pakes A., 1986. Estimates of the value of Patent Rights in European Countries During the Post-1950 Period. The Economic Journal, 96(384), 1052-76.

[32] Schwartz E.S., 2004. Patents and R\&D as real options, Economic Notes, 33(1), 23-54. 
[33] Schwartz E.S., Moon M., 2000. Evaluating Research and Development Investment, in Brennan and Trigeorgis (eds), Innovation, Infrastructure and Strategic Options New York: Oxford University Press, 85-106.

[34] Stoyan, D., Kendall, W.S., Mecke, J., 1995. Stochastic Geometry and its Applications. Chichester: John Wiley \& Sons.

[35] Weeds H., 2002. Strategic Delay in a Real Options Model of R\&D Competition. Review of Economic Studies, 69(3), 729-747.

\section{Appendix: some theory on Spatial Mixed Pois- son Processes}

This Appendix contains the definition and some recent results on SMPPs, that can be already found in the literature.

Let us define a probability space $(\Omega, \mathcal{F}, P)$ containing all the random variables involved in our discussion.

SMPPs are particular spatial point processes. For a survey of the general theory of spatial point processes, we remind to Daley and Vere-Jones (1988) and Stoyan et al. (1995).

Let us consider a measure space $\left(\mathbb{R}^{k}, \mathcal{B}\left(\mathbb{R}^{k}\right), M\right)$, where $\mathcal{B}\left(\mathbb{R}^{k}\right)$ is the Borel $\sigma$-algebra and $M$ is absolutely continuous with respect to the Lebesgue measure. We also introduce a nonnegative random variable $\Lambda$ with probability distribution $U: \mathbb{R} \rightarrow[0,1]$.

Definition 1. A spatial process $R$ is Mixed Poisson with mixing distribution $U$ and baseline intensity measure $M(\cdot)$ if and only if, for $I \in \mathcal{B}\left(\mathbb{R}^{k}\right)$ and for $n \in \mathbb{N}$,

$$
P(R(I)=n)=\int_{0}^{\infty} e^{-\lambda M(I)} \frac{[\lambda M(I)]^{n}}{n !} d U(\lambda) .
$$

We need some properties on the SMPPs, that have been used in our work. Since $M$ is absolutely continuous with respect to the Lebesgue measure, the following result states immediately.

Lemma 1. A SMPP is a simple point process.

Now, consider a spatial point process $R \equiv\left\{X_{i}\right\}_{i \in \mathbb{N}}$, with $X_{i} \in \mathcal{X} \subseteq \mathbb{R}^{k}, k \in \mathbb{N}$, for any $i \in \mathbb{N}$.

Consider a sequence of i.i.d. random variables $\mathbf{W}=\left\{W_{i}\right\}_{i \in \mathbb{N}}$ taking values on a set $\mathcal{W} \subseteq \mathbb{R}^{n}$ for some $n \in \mathbb{N}$.

Define a transformation

$$
\phi: \mathcal{X} \times \mathcal{W} \rightarrow \mathcal{Y} \subseteq \mathbb{R}^{k}
$$

where $\phi(\cdot, w): \mathcal{X} \rightarrow \mathcal{Y}$ is measurable and one-to-one for any fixed $w \in \mathcal{W}$.

We define the transformed spatial point process as follows:

$$
N=\left\{\phi\left(X_{i}, W_{i}\right)\right\}_{i \in \mathbb{N}}
$$


We can also write $N=\Phi_{\phi}(R, \mathbf{W})$ instead of (20).

The following theorem is already known in the literature (see e.g. Cinlar (1995)) and a proof based on stochastic geometrical arguments can be found in Foschi and Spizzichino (2008).

Theorem 1. Let $R$ be a SMPP with mixing distribution $U$ and baseline intensity measure $M$. Consider a sequence of i.i.d. random variables $\mathbf{W}=\left\{W_{i}\right\}_{i \in \mathbb{N}}$, with distribution $G$ and independent of $R$.

Then $N=\Phi_{\phi}(R, \mathbf{W})$ is a SMPP with the same mixing distribution $U$ and intensity measure

$$
M^{\star}(J)=\int_{\mathbb{R}^{n}} M\left(\phi_{w}^{-1}(J)\right) d G(w),
$$

where $J \subseteq \mathcal{Y}$ and $X \in \phi_{w}^{-1}(J)$ if and only if $\phi(X, w) \in J$.

Theorem 1 is a key result in our work, since it explains the invariance of SMPPs with respect to a very general class of transformations.

A further result can be provided concerning conditional estimates of SMPPs realizations. Let us consider three regions $I \subseteq \mathcal{X}, J, H \subseteq \mathcal{Y}$. We aim at estimating the number of points of $N$ fallen in $H$, knowing the restriction of the processes $R$ and $N$ to the regions $I$ and $J$ respectively. First of all, we need to introduce a notation describing the number of points of $R$ fallen in $I$ and sent by the transformation $\phi$ into $J$ or into $H$. The latter quantities are represented by the random variables $N_{(I)}(J)$ and $N_{(I)}(H)$ respectively, given by:

$$
N_{(I)}(K) \equiv \sum_{\alpha \in A} \mathbf{1}_{\left\{\phi\left(X_{\alpha}, W_{\alpha}\right) \in K\right\}} \mathbf{1}_{\left\{X_{\alpha} \in I\right\}}, \quad K \in\{J, H\} .
$$

A straightforward computation proves the following result:

Lemma 2. For a given $I \subseteq \mathcal{X}, N_{(I)}$ can be thought of as a SMPP of its own, with mixing distribution $U$ and baseline intensity measure

$$
M_{(I)}^{\star}(J)=\int_{\mathbb{R}^{n}} M\left(I \cap \phi_{w}^{-1}(J)\right) d G(w) .
$$

Define the event:

$$
E_{(l, m)} \equiv\left\{N_{(I)}(H)=l, N_{(I)}(J)=m\right\}, \quad l, m \in \mathbb{N} \cup\{0\} .
$$

In Cerqueti et al. (2009), the following theorem is proved:

Theorem 2. For arbitrary subsets $I, J, H$, we have

$$
\begin{gathered}
P\left(N(H)=n \mid R(I)=n^{\prime}, N(J)=n^{\prime \prime}, E_{(l, m)}\right) \\
=\int_{0}^{\infty} \frac{\left[\lambda M_{(\bar{I})}^{\star}(H)\right]^{n-l}}{(n-l) !} e^{-\lambda M_{(\bar{I})}^{\star}(H)} u\left(\lambda ; I, J, n^{\prime}, n^{\prime \prime}, m\right) d \lambda,
\end{gathered}
$$

where

$$
u\left(\lambda ; I, J, n^{\prime}, n^{\prime \prime}, m\right)=\frac{\lambda^{n^{\prime \prime}-m+n^{\prime}} e^{-\lambda\left[M_{(\bar{I})}^{\star}(J)+M(I)\right]} u(\lambda)}{\int_{0}^{\infty} \lambda^{n^{\prime \prime}-m+n^{\prime}} e^{-\lambda\left[M_{(\bar{I})}^{\star}(J)+M(I)\right]} u(\lambda) d \lambda} .
$$


Theorem 2 also provides an estimate of the parameter $\Lambda$. We notice, in fact, that $u\left(\lambda ; I, J, n^{\prime}, n^{\prime \prime}, m\right)$ coincides with the posterior distribution of $\Lambda$ given the observation of the event $\left\{R(I)=n^{\prime}, N(J)=n^{\prime \prime}, N_{(I)}(J)=m\right\}$, i.e. $u\left(\lambda ; I, J, n^{\prime}, n^{\prime \prime}, m\right)=$ $U\left(\lambda \mid R(I)=n^{\prime}, N(J)=n^{\prime \prime}, N_{(I)}(J)=m\right)$. This fact allows us to explicitly derive the distribution of the transformed process $N$, starting from the definition of the SMPP $R$. 